\title{
Study of the strong decays of $\phi(2170)$ and the future charm-tau factory
}

\author{
Hong-Wei $\mathrm{Ke}^{1, *}$ and Xue-Qian $\mathrm{Li}^{2, \dagger}$ \\ ${ }^{1}$ School of Science, Tianjin University, Tianjin 300072, China \\ ${ }^{2}$ School of Physics, Nankai University, Tianjin 300071, China \\ (Received 30 October 2018; published 19 February 2019)
}

\begin{abstract}
The present data imply that $\phi(2170)$ may not be an excited state of $\phi$ but is a four-quark state with $s s \bar{s} \bar{s}$ constituents. Furthermore, there are no two mesons of $s \bar{s}$ available to form a molecule that fits the mass spectrum of $\phi(2170)$; thus, we suggest it should be an $s s \bar{s} \bar{s}$ tetraquark state. In this scenario, we estimate its decay rates through the fall-apart mechanism. Our theoretical estimates indicate that its main decay modes should be $\phi(2170)$ into $\phi f_{0}(980), h_{1} \eta, h_{1} \eta^{\prime}, K_{1}(1270) K$, and $K_{1}(1400) K$. Under this hypothesis, the modes $\phi(2170) \rightarrow K^{*}(890)^{0} \bar{K}^{*}(890)^{0}, K^{+} K^{-}$, and $K_{L}^{0} K_{S}^{0}$ should be relatively suppressed. Since the width of $h_{1}$ is rather large, at present, it is hard to gain precise data on $\operatorname{BR}\left(\phi(2170) \rightarrow h_{1} \eta\right)$ and $\operatorname{BR}\left(\phi(2170) \rightarrow h_{1} \eta^{\prime}\right)$, the measurements of which may be crucial for drawing a definite conclusion about the inner assignment of $\phi(2170)$. We place our expectations on the proposed charm-tau factory, which will have much larger luminosity and better capacities.
\end{abstract}

DOI: $10.1103 /$ PhysRevD.99.036014

\section{INTRODUCTION}

Very recently, a meson $\phi(2170)$ came into the view of researchers because it may be a special exotic state. It was observed via its decay into $\phi+f_{0}(980)$ [1]; meanwhile, some possible final states $K^{* 0} K^{ \pm} \pi^{ \pm}$and $K^{* 0} \bar{K}^{* 0}$ have not been seen. If it were a normal meson i.e., an excited state of $\phi$ the decay portals into $K^{+} K^{-}$and $K_{L}^{0} K_{S}^{0}$ would be dominant as the ground $\phi$ does. Moreover, even the channel $K^{* 0} \bar{K}^{* 0}$ should also be seen since a sufficient phase space is available. Furthermore, in Ref. [2], the theoretical evaluation on the total width obviously conflicts with data if $\phi(2170)$ is a normal meson. A reasonable interpretation is needed. It is suggested that the observed $\phi(2170)$ could be a molecular state of $\Lambda \bar{\Lambda}[3]$ or a tetraquark state [4]. In Ref. [5], the author thinks that $\phi(2170)$ is an excited $q \bar{q} s \bar{s}$ tetraquark $(q=u, d)$. But this assignment is questionable because no ground $q \bar{q} s \bar{s}$ tetraquark has ever been observed.

Being hinted at by the decay mode $\phi(2170) \rightarrow$ $\phi f_{0}(980)$, a natural conjecture is that $\phi(2170)$ may be a four-quark state with $s s \bar{s} \bar{s}$ constituents. There are two choices: a molecular state or a tetraquark. However, between two mesons, there should exist a binding energy of about a few tens of mega-electron-volts [6-9]; thus, we

\footnotetext{
*khw020056@hotmail.com

†lixq@nankai.edu.cn
}

Published by the American Physical Society under the terms of the Creative Commons Attribution 4.0 International license. Further distribution of this work must maintain attribution to the author(s) and the published article's title, journal citation, and DOI. Funded by SCOAP . cannot find two available mesons [1] with $s \bar{s}$ constituents to form a molecular hadron of which the mass fits the mass spectrum of $\phi(2170)$. Thus, we turn to suggest that it is an $s s \bar{s} \bar{s}$ tetraquark state. This conjecture was also considered by the authors of Ref. [10].

At the end of last century, a stimulating question was raised: did multiquark states indeed exist in nature, because in their primary paper Gell and Mann predicted them along with the simplest assignments of $q \bar{q}$ for mesons and $q q q$ for baryons [11]. The first proposed pentaquark of $q q q q \bar{s}$ with unusual $B=1$ and $S=1$ quantum numbers would definitely be a multiquark state. In that assignment of the pentaquark $q q q q \bar{s}$ except $\bar{s}$, all other quarks are light ones ( $u$ or $d$ types). The passion of detecting such pentaquarks was very high; however, after a hard and desperate search, such pentaquarks were never observed experimentally. The despair discouraged researchers, who decided to give up. But following conduction of more accurate experiments and innovated skills of analysis, many exotic mesons have been measured. These exotic states are proposed to be four-quark states (molecular states or tetraquarks states) [12-21], later two pentaquarks were observed by the LHCb Collaboration [22], which validates the suggestion about the existence of multiquark states. It validates the suggestion about the existence of multiquark states. However, we have observed that all the discovered multiquark states contain at least one heavy quark ( $c$ or $b$ ). This may hint that the existence of heavy quarks in the multiquark states is fatal [23]. Is that the conclusion of the story? $\phi(2170)$, which has come to our attention recently, could be identified as a four quark state with $s s \bar{s} \bar{s}$ constituents. Even though it is true, the early allegation might not be 
completely subverted because the mass of the $s$ quark resides between that of a very light quark and the supposed "heavy" charm quark and the remaining constituents in the exotic state are all $s$ flavor $(\bar{s})$ with "middle" mass.

A naive analysis may provide us support for this conjecture. The masses of $\Omega$ and $\phi$, which consist of three $s$ quarks and an $s \bar{s}$, respectively, are 1672 and $1020 \mathrm{MeV}$. This implies that the $s$-quark mass is around $500-600 \mathrm{MeV}$; thus, a simple estimate on the mass of the $s s \bar{s} \bar{s}$ tetraquark state should fall in a region close to the mass of $\phi(2170)$. If the assignment is true, $\phi(2170)$ is indeed a tetraquark with a single flavor of strangeness.

Without doubt, it is absolutely important to get a better understanding of the inner structure of $\phi(2170)$. Since it only possesses $s$ flavor, its decays would be dominated by the modes in which the final states mainly contain $s$ flavors. Let us turn to investigate the mechanism that governs the strong decay of $\phi(2170)$. It is the so-called fall-apart mechanism [24,25].

In Refs. [24,25], the authors suggested that the fall-apart mechanism induces the main decay modes of the tetraquark state. By this mechanism, the constituents in a tetraquark are rearranged into two color singular pairs by exchanging soft gluons and then simply fall apart into two mesons. In this work, we will employ this mechanism to study the decays of $\phi(2170)$.

This paper is organized as follows. After the Introduction, in Sec. II, we will explore the decays of $\phi(2170)$. Section III is devoted to our conclusion and discussions.

\section{FALL-APART DECAYS OF $\phi(2170)$}

Since $\phi(2170)$ of $J^{P}=1^{-}$is supposed to be an $s s \bar{s} \bar{s}$ tetraquark, which is in a diquark-antiquark configuration, its spin state is

$$
\left|J, J_{12}, J_{34}\right\rangle=|1,1,1\rangle,
$$

where $J$ is the spin of the tetraquark $s s \bar{s} \bar{s}, J_{12}$ is the spin of $s s$, and $J_{34}$ is the spin of $\bar{s} \bar{s}$. The wave function (colorflavor-spin orbit) of the two strange quarks in the diquark must be totally antisymmetric; for the color-spin there are two possibilities: one is that the two $s$ quarks reside in a color antitriplet and their total spin is 1 (constituting a vector), whereas they can also be in a color sextet with the total spin zero. In a regular baryon, since the third quark is in the color triplet, only one choice remains; i.e., ss should be in an antitriplet to guarantee the baryon is in the color singlet, so the total spin of ss must be 1 . However, in a tetraquark the light diquark with an $[s s]$ content may be in a color-sextet while the other diquark with $\bar{s} \bar{s}$ content in an anti-sextet, in that case, the spin of the $s s$ system can be zero i.e., it is a scalar or pseudoscalar. But since in the color-sextet state the QCD interaction between two constituents is repulsive, even though such a sextet configuration is not strictly forbidden, it is not favorable. The situation was discussed by Jaffe in Ref. [26], so we also omit the color-sextet state of a diquark. The orbital angular momentum between the diquark and antidiquark is 1, i.e., in the $p$ wave for guaranteeing the parity is negative. $\phi(2171)$ is a spin-1 state $\left|J, J_{12}, J_{34}\right\rangle=|1,1,1\rangle$, which has three spin projections: $\left|J, J_{z}\right\rangle=|1,1\rangle,,|1,0\rangle,,|1,-1$,$\rangle .$ Since the $C$ parity of $\phi(2170)$ is odd the spin configuration of the tetraquark is fully determined, for example,

$$
|1,1\rangle=\frac{1}{\sqrt{2}}\left(|1,1\rangle_{s s}|1,0\rangle_{\bar{s} \bar{s}}-|1,0\rangle_{s s}|1,1\rangle_{\bar{s} \bar{s}}\right) .
$$

The color configuration is $|1, \overline{3}, \overline{3}\rangle$, which can be written as [25]

$$
\frac{1}{\sqrt{48}} \varepsilon_{a b d} \varepsilon^{a e f}\left(s^{b} s^{d}\right)\left(\bar{s}_{e} \bar{s}_{f}\right) .
$$

Note the spin configuration of the tetraquark $s s \bar{s} \bar{s}$ is in the diquark and antidiquark spin bases. When it decays via the fall-apart mechanism, one needs to switch a pair quarkantiquark around and rearrange their spins and colors to make proper combinations for the two mesons in the final state.

Now, let us study the decay of $\phi(2170)$ via the fall-apart mechanism. Apparently, the two-body final states with the $s$ wave are preferred if it is allowed. Since the $J^{P C}$ of $\phi(2170)$ is a $1^{--}$tetraquark, $s s \bar{s} \bar{s}$ can fall apart into two mesons with the quantum number assignments as $1^{--}$and $0^{++}$or $1^{+-}$and $0^{-+}$,

$$
\begin{aligned}
\left|1, J_{z}\right\rangle= & \frac{1}{2}\left(|1, m\rangle_{13}|0,0\rangle_{24}+|0,0\rangle_{13}|1, m\rangle_{24}\right. \\
& \left.+|1, m\rangle_{14}|0,0\rangle_{23}+|0,0\rangle_{14}|1, m\rangle_{23}\right),
\end{aligned}
$$

with $J_{z}=m$.

$\phi(2170)$ can also fall apart into two mesons with the quantum numbers $1^{++}$and $1^{--}$,

$$
\begin{aligned}
\left|1, J_{z}\right\rangle= & \frac{1}{\sqrt{2}}\left(\sum_{m_{13} m_{24}} C_{m_{13} m_{24}}\left|1, m_{13}\right\rangle\left|1, m_{24}\right\rangle\right. \\
& \left.+\sum_{m_{14} m_{23}} C_{m_{14} m_{23}}\left|1, m_{14}\right\rangle\left|1, m_{23}\right\rangle\right),
\end{aligned}
$$

with $\mathbf{J}_{13}=\mathbf{J}_{1}+\mathbf{J}_{3}, \mathbf{J}_{24}=\mathbf{J}_{2}+\mathbf{J}_{4}, m_{13}$ and $m_{24}$ being their projections along the $\mathrm{Z}$ axis, and $J_{z}=m_{13}+m_{24}$. $C_{m_{13} m_{24}}$ and $C_{m_{14} m_{23}}$ are corresponding Clebsch-Gordan coefficients.

One also notices that the $I^{G}$ of $\phi(2170)$ is $0^{-}$, so for such strong Okubo-Zweig-lizuka allowed decays the two final mesons should more favorably be in $I^{G}=0^{-}$and $0^{+}$ respectively. Of course, the combination of $1^{-}, 1^{+}$could also work, but naively may be suppressed (further discussion will be presented in the last section). This analysis advocates the final states $\phi(1020) f_{0}(980), \quad \phi(1020) f_{0}(500)$, $\phi(1680) f_{0}(500), \quad \omega(782) f_{0}(980), \quad \omega(782) f_{0}(500)$, $\omega(1420) f_{0}(500), \quad \omega(1650) f_{0}(500), \quad h_{1}(1170) \eta$, 
TABLE I. Some factors for the decay $\phi(2170) \rightarrow$ two mesons.

\begin{tabular}{lccccccccc}
\hline \hline Decay mode & $\phi f_{0}(980)$ & $\phi f_{0}(500)$ & $h_{1} \eta$ & $h_{1} \eta^{\prime}$ & $\phi \eta$ & $\phi \eta^{\prime}$ & $\omega f_{0}(980)$ & $K_{1}(1270) K$ & $K_{1}(1400) K$ \\
\hline Color factor & $\frac{2}{\sqrt{3}}$ & $\frac{2}{\sqrt{3}}$ & $\frac{2}{\sqrt{3}}$ & $\frac{2}{\sqrt{3}}$ & $\frac{2}{\sqrt{3}}$ & $\frac{2}{\sqrt{3}}$ & $\frac{2}{\sqrt{3}}$ & $\frac{4}{3 \sqrt{3}}$ & $\frac{4}{3 \sqrt{3}}$ \\
Spin factor & $2 \sqrt{2}$ & $2 \sqrt{2}$ & $2 \sqrt{2}$ & $2 \sqrt{2}$ & $2 \sqrt{2}$ & $2 \sqrt{2}$ & $2 \sqrt{2}$ & 4 & 4 \\
Flavor factor & $c_{1}^{\prime}$ & $c_{2}^{\prime}$ & $c_{1}^{\prime \prime}$ & $c_{2}^{\prime \prime}$ & $c_{1}^{\prime \prime}$ & $c_{2}^{\prime \prime}$ & $c_{1}^{\prime}$ & 1 & 1 \\
Phase space factor $^{\mathrm{a}}$ & 0.0036 & 0.0056 & 0.0054 & 0.0018 & 0.0062 & 0.0038 & 0.0053 & 0.0050 & 0.0041 \\
\hline \hline
\end{tabular}

${ }^{\mathrm{a}}$ The partial decay width is $d \Gamma=\frac{1}{32 \pi^{2}} \frac{\mid \mathbf{p}}{m^{2}}|\mathcal{M}|^{2} d \Omega$, where $\mathcal{M}$ is the hadronic transition amplitude. Supposing it is irrelevant to the solid angle, one can easily integrate the width over the phase space factor $\frac{1}{8 \pi} \frac{|\mathbf{p}|}{\mathrm{m}^{2}}$.

$h_{1}(1170) \eta^{\prime}$, and $\omega(782) f_{1}(1285)$, which satisfy all the constraints from matching concerned quantum numbers.

In the simple quark model, $(s \bar{s})_{1^{--}}$can be decomposed into $c_{1} \phi(1020)+c_{2} \omega(782)$, where the values of $c_{1} \simeq 1$ and $c_{2} \simeq 0$ are estimated by fitting the decay rates of $\phi(1020) \rightarrow K^{+} K^{-}$and $\phi(1020) \rightarrow \pi^{+} \pi^{-}[1]$. In this picture, $\omega$ only contains a very tiny fraction of strange flavor; thus, those modes involving $\omega(782)$ in the aforementioned channels would have a very small probability of occurring via the fall-apart mechanism directly, but the channel $\phi(2170) \rightarrow f_{0}(980) \omega$ still has a chance to be measured, which we will discuss latter.

If $f_{0}(980)$ and $f_{0}(500)$ are two normal mesons [27-31], the decomposition follows $(s \bar{s})_{0^{++}}=c_{1}^{\prime} f_{0}(980)+$ $c_{2}^{\prime} f_{0}(500)$. Moreover, another relation is $(s \bar{s})_{0^{-+}}=$ $c_{1}^{\prime \prime} \eta+c_{2}^{\prime \prime} \eta^{\prime}$. For the $1^{+-}$quantum system, the only candidate is $(s \bar{s})_{1^{+-}}=h_{1}(1170)$. With those decompositions, we may estimate the corresponding decay rates of $\phi(2170)$ into the final products involving those mesons via the fallapart mechanism.

It is widely accepted that if the fall-apart mechanism exists the dominant decay processes should be determined via this mechanism. Thus, we can estimate the decay rates of $\phi(2170)$ roughly by inputting the coefficients of relevant decompositions, and the relations are listed in the following Table I.

Relevant factors for the decays of $\phi(2170)$ are listed in Table I. There exists an unknown factor $g_{F A}$ that is the parameter corresponding to the fall-apart mechanism, and it should be universal for all the processes.

At present, accurate values of the coefficients $c_{1}^{\prime}, c_{2}^{\prime}, c_{1}^{\prime \prime}$, and $c_{2}^{\prime \prime}$ cannot be obtained from data because, so far, there are no measurements with sufficient precision on the relevant processes available. However, we can make rough estimates using the information we have so far. That is what we are doing below.

$\phi \rightarrow f_{0}(980) \gamma$ exists, but $\phi \rightarrow f_{0}(500) \gamma$ does not [1]; this fact implies $c_{1}^{\prime} \simeq 1$. Of course, a possibility is that $f_{0}(500)$ is a rather wide resonance, and such a radiative decay would be hard to observe. Anyhow, one can roughly assert that the probability of finding the channel $\phi(2170) \rightarrow \phi f_{0}(500)$ may be small, and then we set $c_{1}^{\prime}=1$.
Both $\Gamma\left(D_{s} \rightarrow \eta^{\prime} \pi^{+}\right)$and $\Gamma\left(D_{s} \rightarrow \eta \pi^{+}\right)$have been measured, and one can obtain the ratio of the rates of the two channels as $\Gamma\left(D_{s} \rightarrow \eta^{\prime} \pi^{+}\right) / \Gamma\left(D_{s} \rightarrow \eta \pi^{+}\right)=2.32$. Taking into account the phase space difference 0.82 , we obtain the ratio $c_{2}^{\prime \prime} / c_{1}^{\prime \prime}=1.68$. Then, we can use the ratio and the required normalization condition $\left|c_{1}^{\prime \prime}\right|^{2}+\left|c_{2}^{\prime \prime}\right|^{2}=1$ to determine the modules of coefficients $c_{1}^{\prime \prime}$ and $c_{2}^{\prime \prime}$. The advantage of using the ratio instead of the widths enables us to avoid some experimental errors. Finally, $c_{2}^{\prime \prime}=0.89$ and $c_{1}^{\prime \prime}=$ 0.51 are achieved. However, in this scenario, we cannot determine the relative phase between $c_{1}^{\prime \prime}$ and $c_{2}^{\prime \prime}$. Using these values, an estimate on the ratios is made as $\Gamma\left(\phi(2170) \rightarrow \phi f_{0}(980)\right): \Gamma\left(\phi(2170) \rightarrow h_{1} \eta\right): \Gamma(\phi(2170) \rightarrow$ $\left.h_{1} \eta^{\prime}\right) \simeq 1: 0.4: 0.4$. We suggest experimentally searching the two channels $\phi(2170) \rightarrow h_{1} \eta$ and $\phi(2170) \rightarrow h_{1} \eta$ because they do have substantial branching ratios and should be "seen" according to our prediction.

Even though $\omega f_{0}(980)$ cannot be produced via the fallapart mechanism, the $s$ from the diquark and $\bar{s}$ from the antidiquark of the tetraquark $s s \bar{s} \bar{s}$ can annihilate into $u \bar{u}$ or $d \bar{d}$, while the remaining $s \bar{s}$ pair forms $f_{0}(980)$ (see Fig. 1); by this picture, $\phi(2170) \rightarrow \omega f_{0}(980)$ still can be seen in the experiment, but comparing with $\phi(2170) \rightarrow \phi f_{0}(980)$, the mode should be suppressed by $\alpha_{s}^{2}$ and an additional color matching factor.

It is noted that transition $D_{s} \rightarrow \omega \pi^{+}$occurs via weak interaction, namely, the charm quark turns into $s+u \bar{d}$, and the spectator $\bar{s}$ joins the produced $s$ quark; thus, the $s \bar{s}$ pair annihilates into $u \bar{u}$ or $d \bar{d}$. Because of the similarity, phenomenologically, we can use the data of $D_{s} \rightarrow \phi \pi^{+}$ and $D_{s} \rightarrow \omega \pi^{+}$to predict the width of $\phi(2170) \rightarrow$ $\omega f_{0}(980)$. Using the ratio $\Gamma\left(D_{s} \rightarrow \omega \pi^{+}\right) / \Gamma\left(D_{s} \rightarrow \phi \pi^{+}\right)=$ 0.053 and taking the corresponding phase factors into

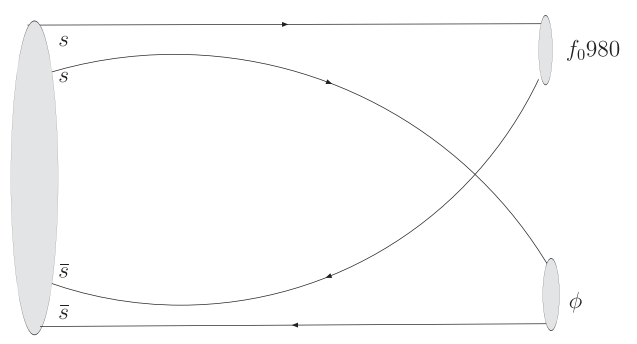

FIG. 1. The Feynman diagram for $s s \bar{s} \bar{s} \rightarrow \phi f_{0}(980)$ transitions via the fall-apart mechanism. 


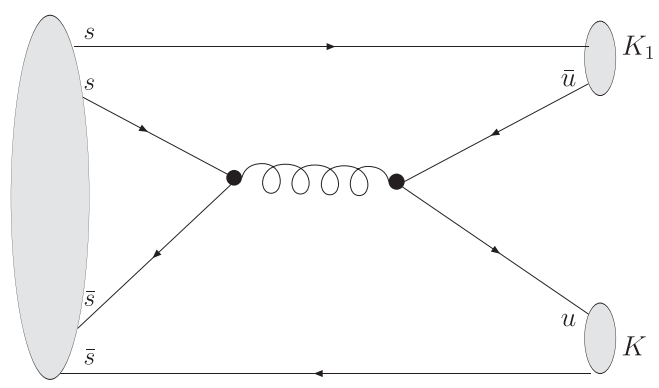

FIG. 2. The Feynman diagram for $s s \bar{s} \bar{s} \rightarrow K_{1} \bar{K}$ transitions.

account, we have $\Gamma\left(\phi(2170) \rightarrow \omega f_{0}(980)\right): \Gamma(\phi(2170) \rightarrow$ $\left.\phi f_{0}(980)\right) \approx 0.068: 1$.

Along the same line, since there are no valence $u \bar{u}$ or $d \bar{d}$ components in the tetraquark $s s \bar{s} \bar{s}, \phi(2170)$ cannot fall apart into $K_{1}(1270) K$ or $K_{1}(1400) K$. To produce $K_{1}(1270) K$ or $K_{1}(1400) K$, an $s \bar{s}$ pair in the tetraquark annihilates into $u \bar{u}$ or $d \bar{d}$. The leading Feynman diagram is Fig. 2. The color and spin factors are presented in Table I, and the production process is somehow similar to $\phi(2170) \rightarrow \phi f_{0}(980)$ but is suppressed by $\alpha_{s}^{2}$. For the $c \bar{c}$ system, $\alpha_{s}$ is about 0.39 [32], whereas for the $s \bar{s}$ case, $\alpha_{s}$ may be slightly larger, but the suppression exists. Moreover, there are double quark-rearrangements (at initial and final sides); thus, an extra factor $g_{F A}$ is introduced.

If we set $\alpha_{s} \sim 0.5$ and $g_{F A} \sim 1$, we expect $\Gamma(\phi(2170) \rightarrow$ $\left.K_{1}(1270) K\right): \Gamma\left(\phi(2170) \rightarrow \phi f_{0}(980)\right) \sim 0.31: 1$. In terms of $\Gamma(\Upsilon(4 S) \rightarrow \Upsilon(1 S) \pi \pi) \approx \Gamma(\Upsilon(4 S) \rightarrow \Upsilon(2 S) \pi \pi)$, we estimate $\Gamma\left(\phi(2170) \rightarrow K_{1}(1270) K\right)$ to be close to $\Gamma\left(\phi(2170) \rightarrow K_{1}(1400) K\right)$. We should ask whether $K^{*}(890)^{0} \bar{K}^{*}(890)^{0}, K^{+} K^{-}$, and $K_{L}^{0} K_{S}^{0}$ can be experimentally measured. Since the relative orbital angular momentum between the daughter mesons is $l=1$, then, because the reactions occur near the threshold, the 3-momentum is small; thus, the $p$-wave suppression will remarkably reduce the production rate, compared to the $s$-wave case. A rough estimate of the suppression factor is $\frac{\mathbf{p}^{2}}{m^{2}} \sim 0.08$. Moreover, to take into account additional factors that may affect evaluation, we adopt the suppression factor for the $p$ wave using the data $\Gamma\left(\psi(2 S) \rightarrow \eta_{c} \gamma\right)$ and $\Gamma\left(\psi(2 S) \rightarrow \chi_{0} \gamma\right)$ and where the 3-momentum of final mesons is close to that in $\phi(2170) \rightarrow K^{*}(890)^{0} \bar{K}^{*}(890)^{0}$. With the ratio $\Gamma(\psi(2 S) \rightarrow$ $\left.\eta_{c} \gamma\right) / \Gamma\left(\psi(2 S) \rightarrow \chi_{0} \gamma\right)$, the production rate of $p$ wave in the case of $\phi(2170)$ is suppressed and is about 0.034 times smaller than that for the $s$ wave. Thus, $\Gamma(\phi(2170) \rightarrow$ $\left.K^{*}(890)^{0} \bar{K}^{*}(890)^{0}\right): \Gamma\left(\phi(2170) \rightarrow \phi f_{0}(980)\right)$ is estimated as $\sim 0.01: 1$. Meanwhile, $\Gamma\left(\phi(2170) \rightarrow K^{+} K^{-}\right)$and $\Gamma\left(\phi(2170) \rightarrow K_{L}^{0} K_{S}^{0}\right)$ should be close to $\Gamma(\phi(2170) \rightarrow$ $\left.K^{*}(890)^{0} \bar{K}^{*}(890)^{0}\right)$.

For other $p$-wave decays of $\phi(2170)$ into $\phi \eta$ and $\phi \eta^{\prime}$ incorporating the phase factors, we estimate $\Gamma(\phi(2170) \rightarrow$ $\phi \eta): \Gamma\left(\phi(2170) \rightarrow \phi \eta^{\prime}\right): \Gamma\left(\phi(2170) \rightarrow \phi f_{0}(980)\right) \sim 0.015:$ $0.025: 1$.

$f_{0}(980)$ may also be considered as a molecular state [33] or a tetraquark [34-36]; if so, the picture would be slightly

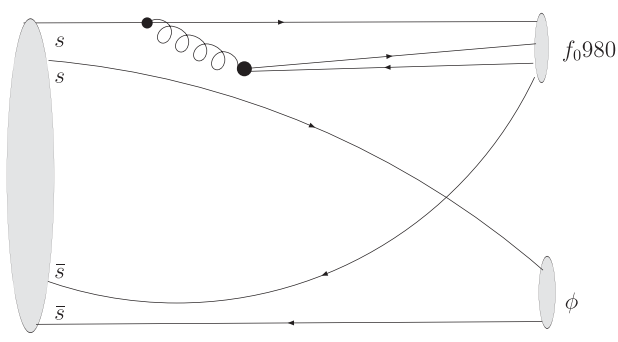

FIG. 3. The Feynman diagram for $s s \bar{s} \bar{s} \rightarrow \phi f_{0}(980)$ transition as $f_{0}(980)$ is regarded as a multiquark state.

different, and the relevant Feynman diagram is shown in Fig. 3. In this case, $\phi(2170) \rightarrow \phi f_{0}(980)$ is suppressed by $\alpha_{s}^{2}$ compared to the aforementioned case in which $f_{0}(980)$ is supposed to be a normal meson. Now, the ratio $\Gamma\left(\phi(2170) \rightarrow \phi f_{0}(980)\right): \Gamma\left(\phi(2170) \rightarrow h_{1} \eta\right)$ : $\Gamma\left(\phi(2170) \rightarrow h_{1} \eta^{\prime}\right)$ is close to $1: 1.6: 1.6$. However, as is well understood, $f_{0}(980)$ may be a mixture of the $s \bar{s}$ state and a multiquark state; thus, according to our estimate, one can roughly evaluate the fraction of each constituent, and this would answer a long-standing question about the identity of $f_{0}(980)$. Obviously, precise measurement on $\phi(2170) \rightarrow \phi f_{0}(980)$ would be very helpful.

At last, we can estimate the results if $\phi(2170)$ is an excited $q \bar{q} s \bar{s}$ tetraquark $(q=u, d)$ as suggested. Naturally, the following decay portals would dominate the total width of $\phi(2170)$; they are $q \bar{q}+s \bar{s}$ and $q \bar{s}+s \bar{q}$, which can be realized via the fall-apart mechanism. In this case, we expect $\phi(2170) \rightarrow \phi f_{0}(500)$ to be the main decay channel rather than $\phi(2170) \rightarrow \phi f_{0}(980) . \Gamma\left(\phi(2170) \rightarrow K_{1}(1270) \bar{K}\right)$ and $\Gamma\left(\phi(2170) \rightarrow K_{1}(1400) \bar{K}\right)$ should be close to $\Gamma(\phi(2170) \rightarrow$ $\left.\phi f_{0}(500)\right)$. In this case, $\phi(2170) \rightarrow K^{*}(890)^{0} \bar{K}^{*}(890)^{0}$, $\phi(2170) \rightarrow K^{+} K^{-}$, and $\phi(2170) \rightarrow K_{L}^{0} K_{S}^{0}$ only receive a $p$-wave suppression, but not color rearrangement suppressions, different from the aforementioned case.

\section{CONCLUSIONS}

With the study on the multiquark structures going deeper and deeper, many unanswered puzzles in this stimulating field have emerged; namely, sharp contradiction between theoretical prediction and experimental observation reminds us that our understanding of the exotic hadrons is far from satisfactory. For example, many theoretical models confirm the existence of $X(5568)$; however, all experimental collaborations have offered negative reports [37-40] except the D0 Collaboration [41]. To compromise the contradiction between theory and experiment, we suggested that a destructive interference between the molecular state and tetraquark suppressed the concerned decay portals [42]. Even though the molecular state and tetraquark are different configurations composed of four quarks, they have the same overall quantum numbers: spin, parity, etc. Actually, any of them can convert into the other via a color rearrangement (or, say, via a quark-antiquark swap). Therefore, generally, the two 
configurations can mix with each other, and the resultant physical exotic meson would be a mixture of them, i.e., $|\phi\rangle=c_{1}|M\rangle+c_{2}|T\rangle$, where $|\phi\rangle,|M\rangle,|T\rangle$ refer to the exotic meson, molecular state, and tetraquark, respectively. For a decay, the hadronic transition matrix element can be written as $\left\langle f\left|H_{\text {eff }}\right| \phi\right\rangle=c_{1}\left\langle f\left|H_{\text {eff }}\right| M\right\rangle+c_{2}\left\langle f\left|H_{\text {eff }}\right| T\right\rangle$, and when we square the amplitude, an interference term emerges. That is the interference between contributions of the molecular state and tetraquark. The coefficients $c_{1}$ and $c_{2}$ in principle can be complex and are determined by nonperturbative QCD. At present, we cannot obtain them from the underlying theory (the nonperturbative QCD) yet. The interference might be constructive or destructive, and so far, we can only fix them by fitting data, namely, determine if it is destructive via a phenomenological study. Indeed, it is a bold conjecture and needs further verification by both theoretical calculations and more accurate experimental measurements.

The decay modes of $\phi(2170)$ imply that the assignment of being an excited state of $\phi$ is disfavored. Some authors have suggested that it should be an exotic state. More concretely, its mass and decay behaviors hint that it may be an $s s \bar{s} \bar{s}$ tetraquark state. Such a structure is special because it may decay via the so-called fall-apart mechanism into hadrons that possess dominantly strange constituents. Employing the fall-apart mechanism, we estimate the decay modes of $\phi(2170)$, which are supposed to be its dominant portals. If $f_{0}(980)$ is a simple meson with $s \bar{s}$ structure, our estimate shows that $\Gamma\left(\phi(2170) \rightarrow \phi f_{0}(980)\right): \Gamma\left(\phi(2170) \rightarrow h_{1} \eta\right): \Gamma$ $\left(\phi(2170) \rightarrow h_{1} \eta^{\prime}\right) \simeq 1: 0.4: 0.4, \Gamma\left(\phi(2170) \rightarrow \omega f_{0}(980)\right): \Gamma$ $\left(\phi(2170) \rightarrow \phi f_{0}(980)\right) \approx 0.068: 1$, and $\Gamma(\phi(2170) \rightarrow$ $\left.K_{1}(1270) K\right): \Gamma\left(\phi(2170) \rightarrow K_{1}(1400) K\right): \Gamma(\phi(2170) \rightarrow$ $\left.\phi f_{0}(980)\right) \sim 0.31: 0.31: 1$. In this case, $\Gamma(\phi(2170) \rightarrow$ $\left.K^{*}(890)^{0} \bar{K}^{*}(890)^{0}\right), \quad \Gamma\left(\phi(2170) \rightarrow K^{+} K^{-}\right), \quad$ and $\Gamma\left(\phi(2170) \rightarrow K_{L}^{0} K_{S}^{0}\right)$ are suppressed by about two orders compared to $\Gamma\left(\phi(2170) \rightarrow \phi f_{0}(980)\right)$.

If $f_{0}(980)$ is a four-quark state, the decay $\phi(2170) \rightarrow$ $\phi f_{0}(980)$ is suppressed, and the ratio $\Gamma(\phi(2170) \rightarrow$ $\left.\phi f_{0}(980)\right): \Gamma\left(\phi(2170) \rightarrow h_{1} \eta\right): \Gamma\left(\phi(2170) \rightarrow h_{1} \eta^{\prime}\right) \simeq$ would be close to $1: 1.6: 1.6$.

Supposing $\phi(2170)$ is an excited $q \bar{q} s \bar{s}$ tetraquark $(q=u, d), \phi(2170) \rightarrow \phi f_{0}(500), \phi(2170) \rightarrow K_{1}(1270) \bar{K}$, and $\phi(2170) \rightarrow K_{1}(1400) \bar{K}$ are expected to be the main decay channels. Even though $\phi(2170) \rightarrow$ $K^{*}(890)^{0} \bar{K}^{*}(890)^{0}, \phi(2170) \rightarrow K^{+} K^{-}$, and $\phi(2170) \rightarrow$ $K_{L}^{0} K_{S}^{0}$ are $p$-wave suppressed modes, since the 3-momenta for these channels are not too small, they should be observed experimentally.

Along with all other subjects in the hadron physics, a better understanding of the exotic state structure and their production and decay mechanisms is badly needed. We all know that the fundamental theory of strong interaction is $\mathrm{QCD}$; however, the nonperturbative QCD that governs the hadron physics is still not yet understood, so various reasonable phenomenological models are adopted by researchers. The study on exotic states may help us to gain more information about the quark model and nonperturbative QCD. As long as the pure $q \bar{q}$, molecular state, and tetraquark have the same overall quantum numbers, they may exist in a mixture. In other words, as an exotic meson (or baryon) behaves peculiarly, i.e., cannot be explained by the simple $q \bar{q}$ structure, one is tempted to refer to it as an exotic hadron containing four quarks or a hybrid state. For a four-quark state, there are two possible configurations: a molecular state and tetraquark. They could mix to result in a physical meson. The mechanism inducing the mixing is nonperturbative QCD effects which are still not well understood. Even though there are some phenomenological models to approach these effects, in fact, none of them can be fully satisfactory. At present, we should try to collect all useful information by studying relevant physical quantities, the mass spectra, production and decay rates, etc., to "guess" a more reasonable hadronic structure. The more information we collect, the closer to reality we will be. Thus, we should try our best to investigate more "exotic" cases. Moreover, $\phi(2170)$ is a special case that might be made of four strange quarks $(s \bar{s} s \bar{s})$; it would be relevant to more interesting physics. All of the constituents have strangeness 1 or -1 , and the identity might simplify the picture and enable us to learn more about the physics picture that does not show up for the exotic mesons containing various flavors.

We suggest measuring all decay modes of $\phi(2170)$ because the data will inform us of its assignment. If the data can decide $\phi(2170)$ to be an $s s \bar{s} \bar{s}$ tetraquark, just as we mentioned in the Introduction, the existence of multiquark state with only $s$ flavor is confirmed and our scope would be widened.

From our discussion, one can notice that to gain more solid knowledge on the structure of exotic states and concerned dynamics is not easy because many inputs adopted in the computations possess large errors. This means that accurate data are the precondition for drawing definite conclusions. So far, the available facilities cannot offer data with satisfactory accuracy in the energy range of charm; thus, we lay hope in the future charm-tau factory, which is planned to be built in China. Since the luminosity of the new facility would be enhanced by several orders more than that of BEPC II and some new detection technique will be used, we can be optimistic that the quality of data will be much improved and the statistics can reach a very high level. Then, we may renew our computations based on the more accurate data and draw definite conclusion not only about $\phi(2170)$ but also about many four-quark states and pentaquarks.

\section{ACKNOWLEDGMENTS}

One of us (Ke) would like to acknowledge a fruitful discussion with Doctor Wen-Biao Yan. This study was supported by the National Natural Science Foundation of China under Contracts No. 11375128 and No. 11675082. 
[1] M. Tanabashi et al. (Particle Data Group), Phys. Rev. D 98, 030001 (2018).

[2] S. Coito, G. Rupp, and E. van Beveren, Phys. Rev. D 80, 094011 (2009).

[3] Y. Dong, A. Faessler, T. Gutsche, Q. Lu, and V. E. Lyubovitskij, Phys. Rev. D 96, 074027 (2017).

[4] A. Ali and W. Wang, Phys. Rev. Lett. 106, 192001 (2011).

[5] S. Takeuchi and M. Takizawa, Proc. Sci. Hadron 2017, 109 (2018).

[6] C. Y. Wong, Phys. Rev. C 69, 055202 (2004).

[7] L. Roca, J. Nieves, and E. Oset, Phys. Rev. D 92, 094003 (2015).

[8] X. Liu, Z. G. Luo, Y. R. Liu, and S. L. Zhu, Eur. Phys. J. C 61, 411 (2009).

[9] I. W. Lee, A. Faessler, T. Gutsche, and V. E. Lyubovitskij, Phys. Rev. D 80, 094005 (2009).

[10] H. X. Chen, C. P. Shen, and S. L. Zhu, Phys. Rev. D 98, 014011 (2018).

[11] M. Gell-Mann, Phys. Lett. 8, 214 (1964).

[12] K. Abe et al. (Belle Collaboration), Phys. Rev. Lett. 98, 082001 (2007).

[13] S. K. Choi et al. (Belle Collaboration), Phys. Rev. Lett. 94, 182002 (2005).

[14] S. K. Choi et al. (Belle Collaboration), Phys. Rev. Lett. 100, 142001 (2008).

[15] B. Aubert et al. (BABAR Collaboration), Phys. Rev. Lett. 95, 142001 (2005).

[16] M. Ablikim et al. (BESIII Collaboration), Phys. Rev. Lett. 112, 132001 (2014).

[17] M. Ablikim et al. (BESIII Collaboration), Phys. Rev. Lett. 111, 242001 (2013).

[18] M. Ablikim et al. (BESIII Collaboration), Phys. Rev. Lett. 110, 252001 (2013).

[19] M. Ablikim et al. (BESIII Collaboration), Phys. Rev. Lett. 113, 212002 (2014).

[20] Z. Q. Liu et al. (Belle Collaboration), Phys. Rev. Lett. 110, 252002 (2013).
[21] I. Adachi (Belle Collaboration), arXiv:1105.4583.

[22] R. Aaij et al. (LHCb Collaboration), Phys. Rev. Lett. 115, 072001 (2015).

[23] X. Q. Li and X. Liu, Eur. Phys. J. C 74, 3198 (2014).

[24] R. L. Jaffe, Phys. Rev. D 15, 281 (1977).

[25] H. Kim, K. S. Kim, M. K. Cheoun, and M. Oka, Phys. Rev. D 97, 094005 (2018).

[26] R. L. Jaffe, Phys. Rep. 409, 1 (2005).

[27] M. D. Scadron, Phys. Rev. D 26, 239 (1982).

[28] D. Klabucar, D. Kekez, and M. D. Scadron, J. Phys. G 27, 1775 (2001).

[29] H. Y. Cheng, Phys. Rev. D 67, 034024 (2003).

[30] H. W. Ke, X. Q. Li, and Z. T. Wei, Phys. Rev. D 80, 074030 (2009).

[31] H.W. Ke and X.Q. Li, Phys. Rev. D 96, 053005 (2017).

[32] E. Eichten, K. Gottfried, T. Kinoshita, K. D. Lane, and T. M. Yan, Phys. Rev. D 17, 3090 (1978); 21, 313(E) (1980).

[33] J. D. Weinstein and N. Isgur, Phys. Rev. Lett. 48, 659 (1982).

[34] R. L. Jaffe, Phys. Rev. D 15, 267 (1977).

[35] L. Maiani, F. Piccinini, A. D. Polosa, and V. Riquer, Phys. Rev. Lett. 93, 212002 (2004).

[36] G. 't Hooft, G. Isidori, L. Maiani, A. D. Polosa, and V. Riquer, Phys. Lett. B 662, 424 (2008).

[37] R. Aaij et al. (LHCb Collaboration), Phys. Rev. Lett. 117, 152003 (2016); 118, 109904(A) (2017).

[38] A. M. Sirunyan et al. (CMS Collaboration), Phys. Rev. Lett. 120, 202005 (2018).

[39] T. A. Aaltonen et al. (CDF Collaboration), Phys. Rev. Lett. 120, 202006 (2018).

[40] M. Aaboud et al. (ATLAS Collaboration), Phys. Rev. Lett. 120, 202007 (2018).

[41] V. M. Abazov et al. (D0 Collaboration), Phys. Rev. Lett. 117, 022003 (2016).

[42] H. W. Ke and X. Q. Li, Phys. Lett. B 785, 301 (2018). 